\title{
Influence of The Smoothing Parameter in The Re-initialization Equation on The Level Set Interface-capturing
}

\author{
Liu Zhilin ${ }^{1, ~ a}$, Huang Yong ${ }^{1, b^{*}}$ \\ ${ }^{1}$ National Key Laboratory Science and Technology on Aero-Engines, Advanced Aero Engine \\ Collaborative Innovation Center, School of Energy and Power Engineering, \\ Beihang University, Beijing 100191, China \\ azhilinliu86@163.com, byhuang@buaa.edu.cn
}

\section{Keywords: Smoothing parameter, Re-initialization, Level set}

\begin{abstract}
The level set method is frequently used to simulate and capture the moving interface in the molten metal casting and forming field. However, the level set function has to be re-initialized after several time-steps. And influence of the smoothing parameter on numerical accuracy of the moving interface-capturing is mainly investigated in the present work, which existing in the implicit iteration re-initialization equation. Further, a novel smoothing parameter approximation is derived and proposed, which keeping almost the interface location motionless while re-initialization. Several typical cases are compared to simulate with the other smoothing parameter approximations proposed in some relative literatures. To diminish numerical scheme's effect, some high order numerical methods in space and time are employed in the simulation. Results evidently show that the smoothing parameter has a strong impact on simulation accuracy of the moving interface-capturing. Meanwhile, compare with the other approximations, it is found that all results computed with the novel smoothing parameter approximation are best. It can greatly improve capturing accuracy of the moving interface. That's to say, it can dramatically decrease the mass loss and capture the sharp interface with high resolution, especially for moving interface with the complicated topological geometry change.
\end{abstract}

\section{Introduction}

Since it was proposed for the first time in [1] as a Eulerian method, the level set method is frequently used to capture rather than track the moving interface. And it can handle easily the complicated topological geometry change of the moving interface, such as deformation, merging and breakup of the interface. Therefore, it is applied extensively to multiphase flow, combustion, crystal growing, molten metal casting and forming fields, and so on. In this method, a signed distance function is defined and used to represent implicitly the surface, which called as the level set function, indicated with $\varphi$. While $\varphi$ is equal to zero, it represents the interface. However, after several time-steps, the level set function may be not the signed distance function any longer, especially for non-uniform flow field. This will result in incorrect interface-capturing and severe numerical oscillation. Hence, re-initialization of the level set function has to be implemented so as to avoid the above mentioned problems.

That idea was first described by Chopp et al in [2], and Sussam et al [3] constructed a specific partial differential equation to implement re-initialization of the level set function, which being Hamilton-Jacobi equation and solved implicitly with the iteration method. It's so called the implicit iteration method. Subsequently, Peng et al [4] improved slightly the partial differential equation. Generally, the computing time cost is very high for solving iteratively the partial differential equation while re-initialization. The time spent of re-initialization is several times more than that of solving the level set advection equation. For this reason, Junbo Cheng et al [5] constructed a fast algorithm of re-initialization to reduce the computing time because only points adjacent to the interface need to be re-initialized. It greatly increases the numerical efficiency. But with the above described re-initialization procedure of the level set function, a considerable amount of total mass is 
lost. To remedy this drawback, a perturbed Hamilton-Jacobi equation is solved to a steady state to accomplish re-initialization of the level set function, in which effect of the interface's local curvature considered [6]. In addition, Zhiliang Wang et al proposed the other algorithm to accomplish re-initialization through locking the interface positions in [7]. And Xueying Zhang et al [8] located the interface at first by the level set function in the vicinity of the interface, then modified it up to desired results. Except for those, the fast marching method [9] and the distance function smoothing method [10] are also employed to re-initialize the level set function. Yet all these two ways are explicit, for which the interface positions having to be firstly located. In [11], the fast marching method and the implicit iteration method were compared for re-initialization of the level set function. The results showed that the latter was better in the case of the moving interface-capturing, and it is also easier for the implementation.

But for the implicit iteration method, it is very obvious that the smoothing parameter affects greatly the resulting re-initialization of the level set function. Even it may be inducing incorrect result while capturing interface. Therefore, that had been discussed and studied in some literatures, such as $[3,10,12-15]$ and so on. To further know that influence of the smoothing parameter on the level set interface-capturing, the present work analyzes and proposes a novel approximation. Moreover, several typical numerical cases are compared to simulate with some different smoothing parameter approximations to validate advantage of the novel approximation.

\section{Re-initialization of the Level Set Function}

The Re-Initialization Equation. In the level set method, the level set function defined as the signed distance function away from the interface is used to represent the surface. And we have to re-initialize the level set function so as to maintain the property of the signed distance function after time evolution of the level set advection equation. Otherwise it results from the numerical dissipation and dispersion, especially when the level set function is very flat and/or sleep in the vicinity of the interface. That is to say, for the level set function, it should satisfy

$$
|\nabla \varphi|=1
$$

Yet that is ideal. And except for the isolate point, the level set function can be stay well behaved for numerical accuracy in the sense that [4],

$$
0<c \leq|\nabla \varphi| \leq C
$$

for some constants $c$ and $C$. As the matter of fact, it is desirable for the many problems.

For the implicit iteration method, the re-initialization equation of the level set function is written as

$$
\left\{\begin{array}{c}
\varphi_{\tau}=\operatorname{sign}\left(\varphi_{0}\right)(1-|\nabla \varphi|) \\
\varphi(\tilde{x}, 0)=\varphi_{0}
\end{array}\right.
$$

where, $\tau$ is the pseudo time, $\varphi_{0}$ is the initial value of the re-initialization of the level set function, sign is the signed function. For the need of the numerical calculation, the signed function is required to smooth,

$$
\operatorname{sign}_{\varepsilon}\left(\varphi_{0}\right)=\frac{\varphi_{0}}{\sqrt{\varphi_{0}^{2}+\varepsilon^{2}}}
$$

where, $\varepsilon$ indicates the smoothing parameter.

The Smoothing Parameter. For Eq. 3, the size of $\operatorname{sign}_{\varepsilon}\left(\varphi_{0}\right)$ has a significant impact on the iteration speed of gaining steady-state solution. If it is small, that means, the smoothing parameter is great, the iterative solution exhibits slowly convergence. Similarly, if the smoothing parameter is smaller, the convergence speed is faster. So the smoothing parameter affects the re-initialization of the level set function, and further affects the level set interface-capturing.

Suppose that the interface locates between the level set function $\varphi_{i}^{0}$ and $\varphi_{i+1}^{0}$, and $\varphi_{i}^{0}<0<$ 
$\varphi_{i+1}^{0} . s_{i}$ and $s_{i+1}$ denote $\operatorname{sign}_{i}\left(\varphi_{0}\right)$ and $\operatorname{sign}_{i+1}\left(\varphi_{0}\right)$, respectively. After one the time step, we have.

For one-dimension, suppose that the interface locates between the level set function $\varphi_{i}^{0}$ and $\varphi_{i+1}^{0}$, and $\varphi_{i}^{0}<0<\varphi_{i+1}^{0}$. $s_{i}$ and $s_{i+1}$ denote $\operatorname{sign}_{i}\left(\varphi_{0}\right)$ and $\operatorname{sign}_{i+1}\left(\varphi_{0}\right)$, respectively. After one time-step, we have

$$
\begin{aligned}
& \varphi_{i}{ }^{1}=\varphi_{i}{ }^{0}+s_{i} \Delta \tau\left(1-\left|\nabla \varphi_{i}\right|\right) \\
& \varphi_{i+1}{ }^{1}=\varphi_{i+1}{ }^{0}+s_{i+1} \Delta \tau\left(1-\left|\nabla \varphi_{i+1}\right|\right)
\end{aligned}
$$

where, superscript indicates the iteration time and subscript indicates the coordinate, $-1 \leq s_{i}<0$ and $0<s_{i+1} \leq 1$. According to the idea from [15], to stay the interface location motionless while re-initializing, the formula was derived

$$
\frac{\sqrt{\varphi_{i}^{0} \varphi_{i}^{0}+\varepsilon_{i}^{2}}}{\varphi_{i+1}^{0} \varphi_{i+1}^{0}+\varepsilon_{i+1}^{2}}=\frac{1-\left|\nabla \varphi_{i}\right|}{1-\left|\nabla \varphi_{i+1}\right|} .
$$

From Eq. 7, we can notice that the approximation of the smoothing parameter is dependent on the slope of the level set function. Combining Eq. 4 and Eq. 7 and for the numerical practices, where we choose $\varepsilon=|\nabla \varphi|$ as a novel smoothing parameter approximation. And in the next, that is validated with the numerical cases.

The Restrict of the Iteration Time Step. To avoid changing the sign of the level set function in the vicinity of the interface while re-initializing, especially the level set function is very flat and/or steep, namely $|\nabla \varphi| \leq \mathrm{c}$ and/or $|\nabla \varphi| \geq \mathrm{C}$, we have to restrict the iteration time step [4]. For that, there has to be

$$
\varphi_{i}{ }^{1}<0<\varphi_{i+1}{ }^{1}
$$

when, $|\nabla \varphi| \leq 1$, Eq. 8 always holds. And $|\nabla \varphi|>1$, we can get the limitation of the time step, for $k=i$ or $k=i+1$, as follows

$$
\Delta \tau \leq \frac{\left|\varphi_{k}\right|}{\left|s_{k}\right| \cdot|1-| \nabla \varphi_{k}||}
$$

In the present work, for the numerical accuracy and oscillation, Eq. 3 is discretized with the fifth order WENO (weighted essentially non-oscillatory) scheme in space and the third order TVD (total variation diminish) Runge-Kutta method in time. So the restriction condition of the time step can be employed with

$$
\Delta \tau=\frac{1}{2} \frac{|\varphi|}{|s| \cdot|1-| \nabla \varphi||}
$$

\section{Numerical Cases}

Materials. Two typical moving interfaces are simulated in the different velocity fields, respectively, the circle interface (see Fig. 1-A) and the slotted disk interface (see Fig. 1-B).

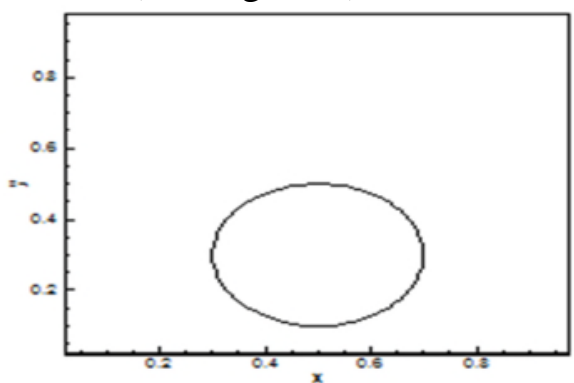

A

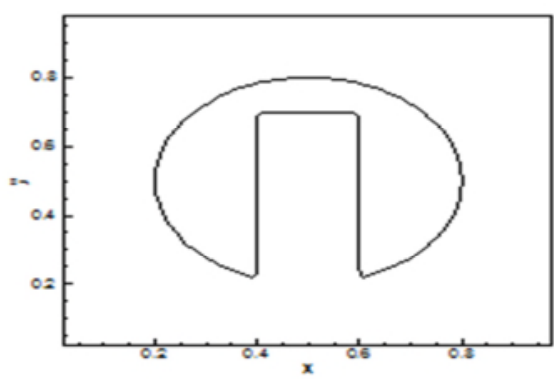

B

Fig. 1 Initial location of the moving interface. 
Deformation of the circle interface is computed in a shearing velocity field and the slotted disk interface is rotated in the rotation velocity field and translated in the translation velocity field. As follows that velocity fields

Shearing

$$
\left\{\begin{array}{l}
u(x, y)=\pi \cdot \cos [\pi(x-0.5)] \cdot \sin [\pi(y-0.5)] \\
v(x, y)=-\pi \cdot \sin [\pi(x-0.5)] \cdot \cos [\pi(y-0.5)]
\end{array}\right.
$$

Rotation

$$
\left\{\begin{array}{l}
u(x, y)=-\pi \cdot(y-0.5) \\
v(x, y)=\pi \cdot(x-0.5)
\end{array}\right.
$$

Translation

$$
\left\{\begin{array}{l}
u(x, y)=0.1 \\
v(x, y)=0.1
\end{array}\right.
$$

Methods. For all cases, the computations are implemented in $50 \times 50$ mesh, calculation domain is $[0,1] \times[0,1]$. And moving the interface with original velocity from $t=0.0$ to $t=1.0$, then going on the calculation with its inverse velocity from $t=1.0$ to $t=2.0$, that is to say, the moving interface return to the initial location in $t=2.0$, where $t$ indicates the evolution time. In the simulation, the different smoothing parameter approximation proposed in different literature will be applied, see Table 1 for details.

Table 1. Approximations of the smoothing parameter.

\begin{tabular}{cccccc}
\hline \multirow{2}{*}{ Smoothing parameter } & \multicolumn{5}{c}{ Approximation proposed in different literature } \\
\cline { 2 - 6 } & {$[12]$} & {$[3,13]$} & {$[10,14]$} & {$[15]$} & present work \\
\hline$\varepsilon$ & $10^{-8}$ & $h$ & $|\varphi| h$ & $\min [(|\nabla \varphi|-|\varphi| / h),|\nabla \varphi|] h$ & $|\nabla \varphi|$ \\
\hline
\end{tabular}

where $h$ is spacing length.

\section{Results and Discussion}

The interface-capturing results in the different velocity field are showed in Fig. 2, of which A, B and $\mathrm{C}$ (the same as below) indicates the shearing, rotation and translation, respectively. Black solid line indicates the original interface, and red, green, blue, purple, brown indicates the interface computed with the smoothing parameter proposed in [15], [10,14], [3,13], [12] and the present work, respectively.

The circle interface performs the behavior of the deformation when it is computed in the shearing velocity, see Fig. 2-A. This kind of deformation is often used to verify the efficiency of the interface-capturing, especially for complicated topology geometry. Hence, we at first compared the interface-capturing accuracy with the smoothing parameters in Table 1 . When $t=1.0$, except for the green and blue interface are almost consistent, there are some great differences between them. Not only the volume contained by the interface become changed, but the sharp-smoothing are also not same. We notice that the purple interface is the least. And by contrast, the brown line calculated with the novel smoothing parameter is the greatest. Further, while the moving interface return to the initial state, namely $t=2.0$, it's very evident that the all resulting interface are smaller than the initial circle, which called the mass loss of the level set interface-capturing, and the trend consists with that when $t=1.0$.

By the same way, the distortion of the moving slotted disk interface is numerically analyzed with its rotation and translation, see Fig. 2-B and Fig. 2-C. results show that all resulting interfaces are less than initial slotted disk interface and the interface sharpness are also smoothed. Compared with results in Fig. 2-A, the differences are not so obvious. Likely, results denoted with the brown line 
are the best. The conclusion is also same as above described. But for topological geometry unchanged, the resulting interfaces are all closer to the initial interface. It can be seen that the smoothing parameter has a strong impact on the mass loss and the sharpness of the level set interface-capturing. Moreover, results computed with the novel smoothing parameter approximation are better than those of the other approximations.

A

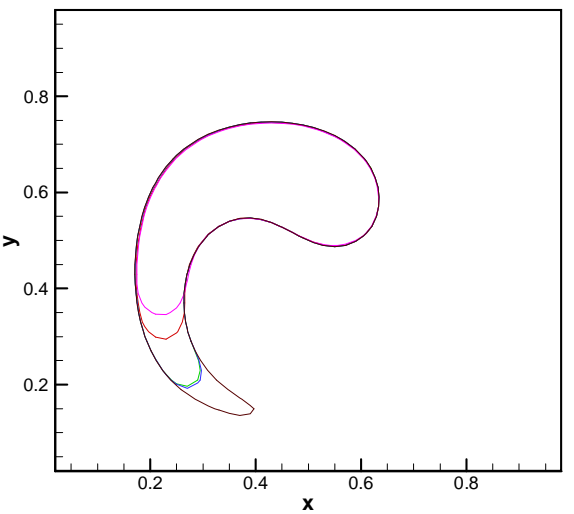

B
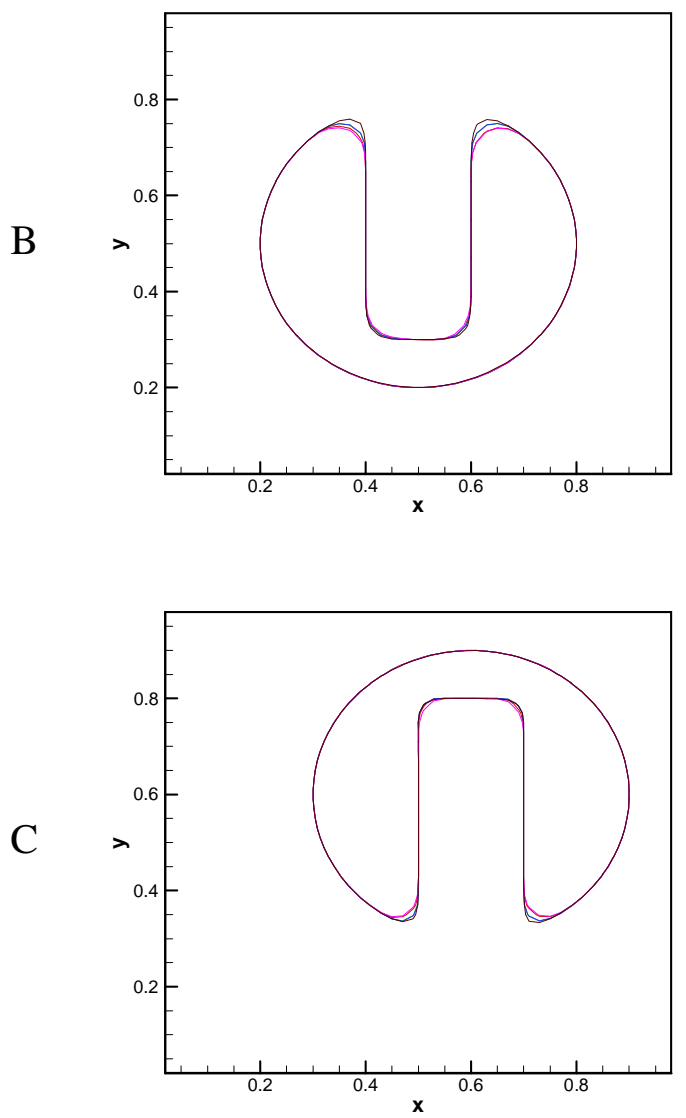

$t=1.0$
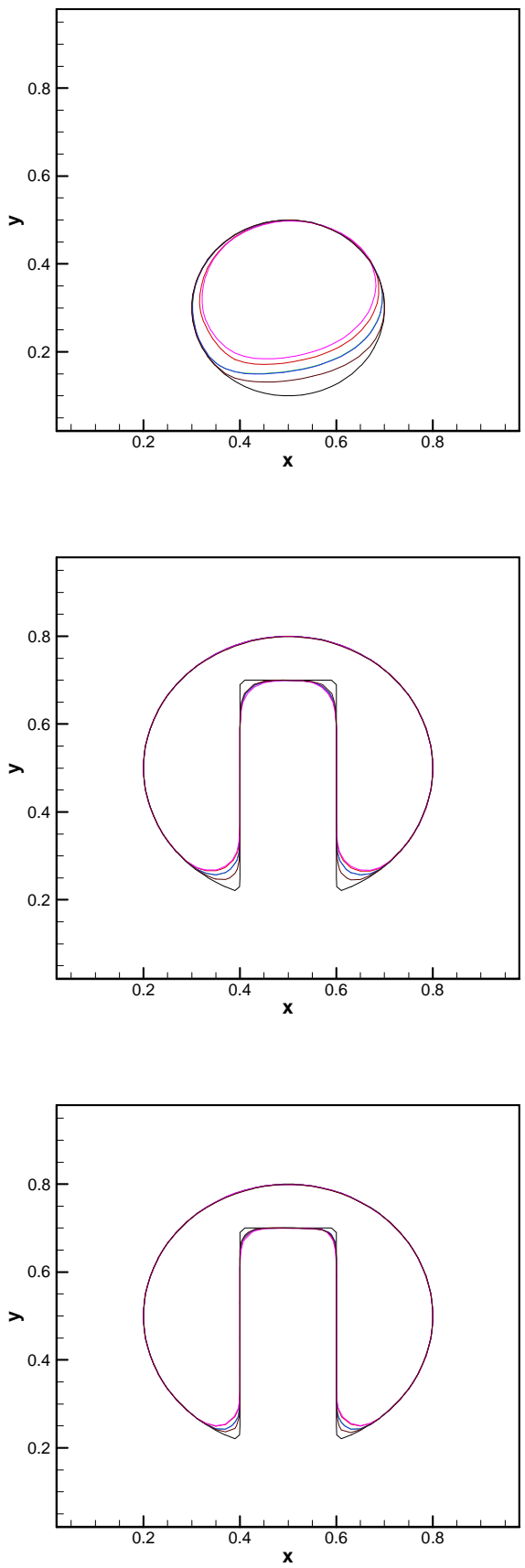

$t=2.0$

Fig. 2 The interface location while $t=1.0$ and $t=2.0$

Fig. 3 shows that mass/volume conservation rate of the resulting interface with different smoothing parameter approximation. Serial number indicate the different smoothing parameter approximation in turn, which proposed in [15], [10,14], [3,13], [12] and the present work, respectively.

It apparently illustrates that effect of the smoothing parameter on the mass conservation of the level set interface-capturing. The result with the novel smoothing parameter approximation is better, 
especially in Fig. 3-A, increasing by 6\%. It means that the novel approximation can conserve well the mass of interface-capturing, this is same as result in Fig. 2.

A
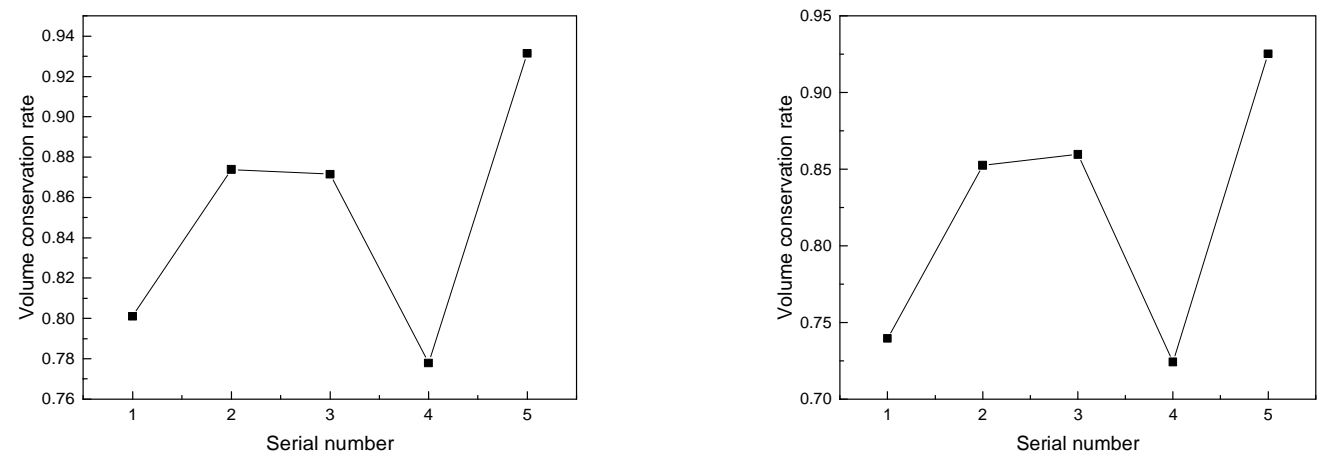

B
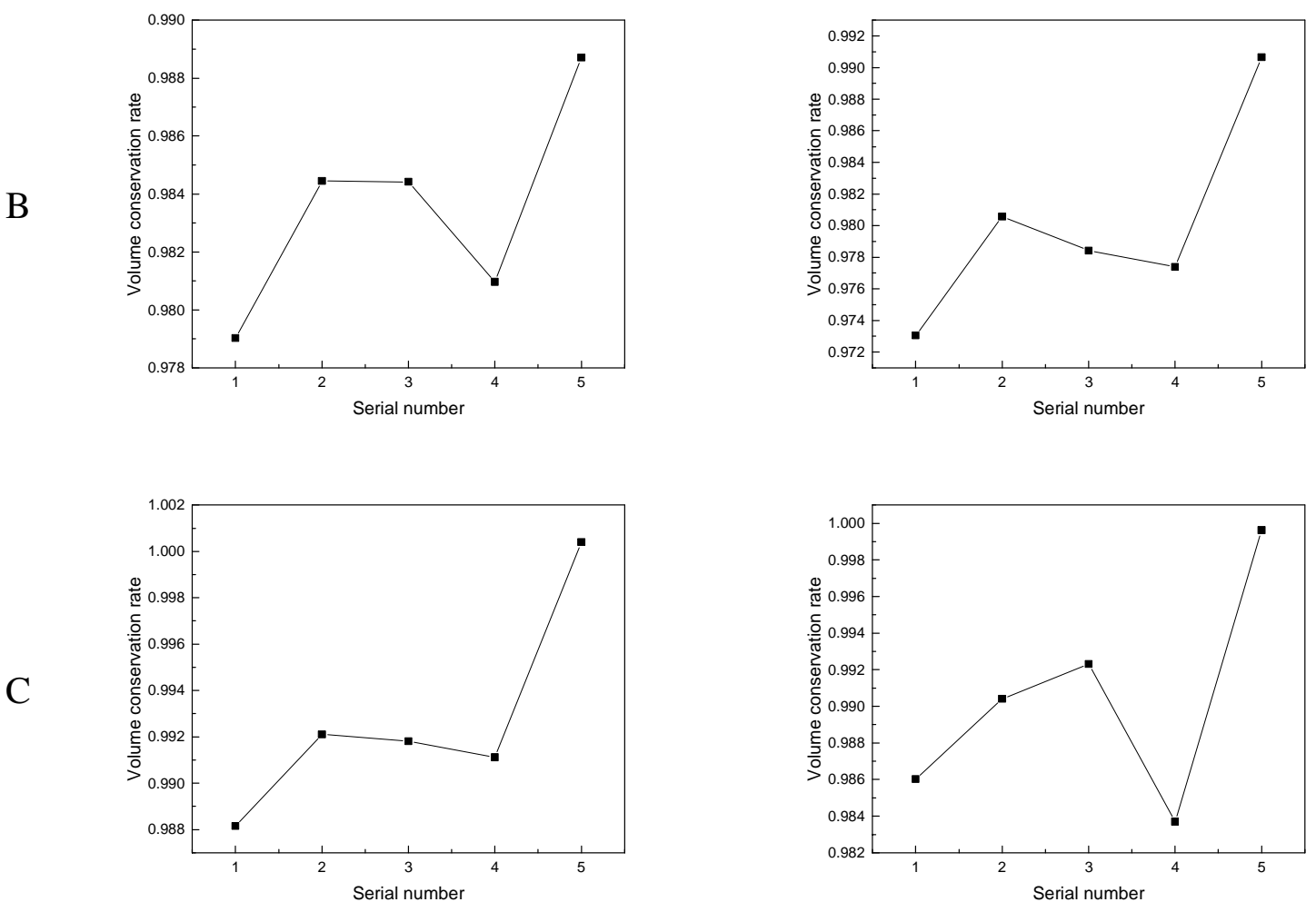

$t=1.0 \mathrm{~s}$

$t=2.0 \mathrm{~s}$

Fig. 3 The comparison of volume conservation rate with different smoothing parameter.

\section{Summary}

In the level set method, the level set function has to be re-initialized after several time-steps, and the smoothing parameter in the implicit re-initialization equation can influence the level set interface-capturing. To gain better numerical results, a novel smoothing parameter approximation is derived and proposed. Two typical moving interfaces are simulated to validate its feasibility and effectiveness, namely, deformation of the circle interface in the shearing velocity field, rotation and translation motion of the slotted disk interface in rotation velocity field and translation velocity field. And the results are compared with those of the other approximations proposed in relative literatures. While computation is implemented, the fifth order WENO scheme in space and the third order TVD Runge-Kutta method in time are employed to discretize the re-initialization equation so as to diminish numerical scheme's effect. Moreover, the iteration time step is also restricted. The results show that the smoothing parameter approximation has a strong impact on accuracy of the level set interface-capturing, such as sharpness and mass conservation of the resulting interface, especially 
for the moving interface with complicated topological geometry change. In addition, compare with the other approximations proposed in some relative literatures, all numerical results computed with the novel smoothing parameter approximation are best. It can greatly improve capturing accuracy of the moving interface. Namely, it can effectively decrease the mass loss and capture the sharp interface with high resolution.

\section{Reference}

[1] S. Osher, J. A. Sethian, Fronts propagating with curvature-dependent speed: Algorithms based on Hamilton-Jacobi formulations. J. Comput. Phy. 79(1) (1988) 12-49.

[2] D. L. Chopp, Computing Minimal Surfaces via Level Set Curvature Flow. J. Comput. Phys. 106(1) (1993) 77-91.

[3] M. Sussman, P. Smereka, S. Osher, A level set approach for computing solutions to incompressible two-phase flow. J. Comput. Phys. 114(1) (1994) 146-159.

[4] D. Peng, et al., A PDE-based fast local level set method. J. Comput. Phys. 155(2) (1999) 410-438.

[5] J. B. Chen, W. J. Tang, Fast algorithm of re-initialization for level set methods. The $12^{\text {th }}$ Symposium for National Hydrodynamics Numerical Methods, The Annual Meeting and The $12^{\text {th }}$ Academic Forum for The Professional Committee of Gas Dynamics Theory, and The Symposium for The Computing Explosion Mechanics. 2005.

[6] Y. C. Chang, et al., A Level Set formulation of eulerian interface capturing methods for incompressible fluid flows. J. Comput. Phys. 124(2) (1996) 449-464.

[7] Z. L. Wang, Z. W. Zhou, An improved level set re-initialization solver. Appl. Math. Mech. (10) (2004) 991-996.

[8] X. Y. Zhang, N. Zhao, An improved re-initialization algorithm for multi-component fluid. Acta Aerodyn. Sin. 24(04) (2006) 438-443.

[9] J. A. Sethian, A fast marching level set method for monotonically advancing fronts. Proc. Nat. Academy Sci. 93(4) (1996) 1591-1595.

[10] L. F. Zhang, et al., A new method for the initialization and reinitialization of level set function. Comput. Eng. Appl. 39(10) (2003) 26-32.

[11] C. Y. Xiao, et al., Fast reconstruction of signed distance function in level set method. Sign. Proc. 19(6) (2003) 551-555.

[12]Q. Yang, et al., An investigation on coupled level-set and VOF algorithm in interface capturing. Aeronaut. Comput. Tech. 04 (2012) 14-19.

[13] W. Zhou, et al., A coupled volume of fluid and level set (CVOFLS) method for tracking moving interfaces. Chinese J. Eng. Math. 32(5) (2015) 697-708.

[14] Y. Zhang, Q. Zou, D. Greaves, Numerical simulation of free-surface flow using the level-set method with global mass correction. Int. J. Numer. Meth. Fluid. 63(6) (2010) 651-680.

[15] B. Zhang, et al., An implicitre-initialization method for level set function keeping interface fixed. Chinese J. Comput. Phys. 28(5) (2011) 667-676. 